\title{
Surgical management of inguinal hernia: retrospective cohort study in southeastern Scotland, 1985-2001
}

H D E Atkinson, S G Nicol, S Purkayastha, S Paterson-Brown

Inguinal hernia repair (IHR) is the most common general surgical procedure in the United Kingdom, with an estimated 80000 cases a year. The past decade has seen an increase in the use of prosthetic mesh in open and laparoscopic hernia repair based on the premise of a tension-free repair. ${ }^{1}$ Conversely, although traditional repair with suturing using the Shouldice technique has been reported with low rates of recurrence, it is difficult to learn and often associated with high rates of recurrence when done in the wider community. Recent reports have indicated that laparoscopic repair is associated with less immediate postoperative pain and a faster return to normal activities, particularly for recurrent and bilateral hernias. ${ }^{2}$ Large case series indicate recurrence rates for open and laparoscopic mesh repairs as low as $0.2 \%,{ }^{3}$ and randomised trials of open primary IHR, with follow up as short as two years, have consistently found fewer recurrences associated with using mesh than not, ${ }^{24}$ with similar results reported for recurrent hernias. ${ }^{5}$ We examined these changing trends in IHR in southeastern Scotland between 1985 and 2001.

\section{Participants, methods, and results}

We retrospectively identified all patients undergoing IHR between January 1985 and January 2002 in six surgical centres in southeastern Scotland using the Lothian surgical audit database (available in all these hospitals for some or all of this period), hospital coding systems (OPCS3 and OPCS4), and data from the Information and Statistics Division of the Public Health Institute of Scotland. Population data were taken from the UK census and the General Register Office for Scotland. Poisson and logistic regression were used to analyse the data.

Overall, 16450 patients had primary IHR (11 274 open suture repairs, 3885 open prosthetic mesh repairs, and 1291 laparoscopic mesh repairs). A further 1859 patients had recurrent IHR (1292 open suture repairs, 338 open mesh repairs, and 229 laparoscopic repairs). In 1993, less than 1\% (8/1324) of all IHRs used mesh, increasing to more than 90\% (1012/ 1114) by 2001 (figure).

Laparoscopic mesh IHR was first introduced to southeastern Scotland in 1993, and its use in primary repair increased to $18 \%(186 / 1027)$ by 1996 , then remained static. For recurrent hernias, however, use continued to rise to $42 \%$ (41/98) by 2001 . Up to this time no surgeon had done prophylactic contralateral IHR in the region.

In this period, the total number of primary repairs increased significantly at an average annual rate of $1.25 \% \quad(\mathrm{P}<0.001)$, while the population within southeastern Scotland remained static at about 745000 residents between 1985 and 1991, and increased by an average $0.49 \%$ a year from 1992 to
2001. The annual number of recurrent repairs did not change significantly, but the annual rate of recurrent repairs decreased significantly as a proportion of the total, from $11.7 \%$ to $8.8 \%(\mathrm{P}<0.001)$. The median patient age at time of surgery did not change significantly over the study period; Spearman's rank correlation $r_{s}=-0.09, P=0.72$ (primary repairs) and $r_{s}=0.35, P=0.16$ (recurrent repairs).

\section{Comment}

The annual ratio of recurrent to primary repairs has significantly decreased in southeastern Scotland, during a period in which surgical technique has changed while selection criteria have remained the same. This ratio started falling before the introduction of mesh repair, indicating that other variables may be involved. Although changes in recurrence rates may lag up to 10 years behind changes in surgical technique, $40 \%$ occur

This article was posted on bmj.com on 16 November 2004: http://bmj.com/cgi/doi/10.1136/bmj.38282.675556.F7

Charing Cross Hospital, London W6 8RF

Department of Orthopaedic and Trauma Surgery

H D E Atkinson specialist registrar Department of Trauma and Orthopaedic Surgery, Bristol Royal Infirmary

S G Nicol specialist registrar Department of General Surgery, St Mary's Hospital, London

S Purkayastha senior house officer

Department of Surgery, Royal Infirmary of Edinburgh

S Paterson-Brown consultant

Correspondence to: H D E Atkinson duscha@hotmail.com

BMJ 2004;329:1315-6
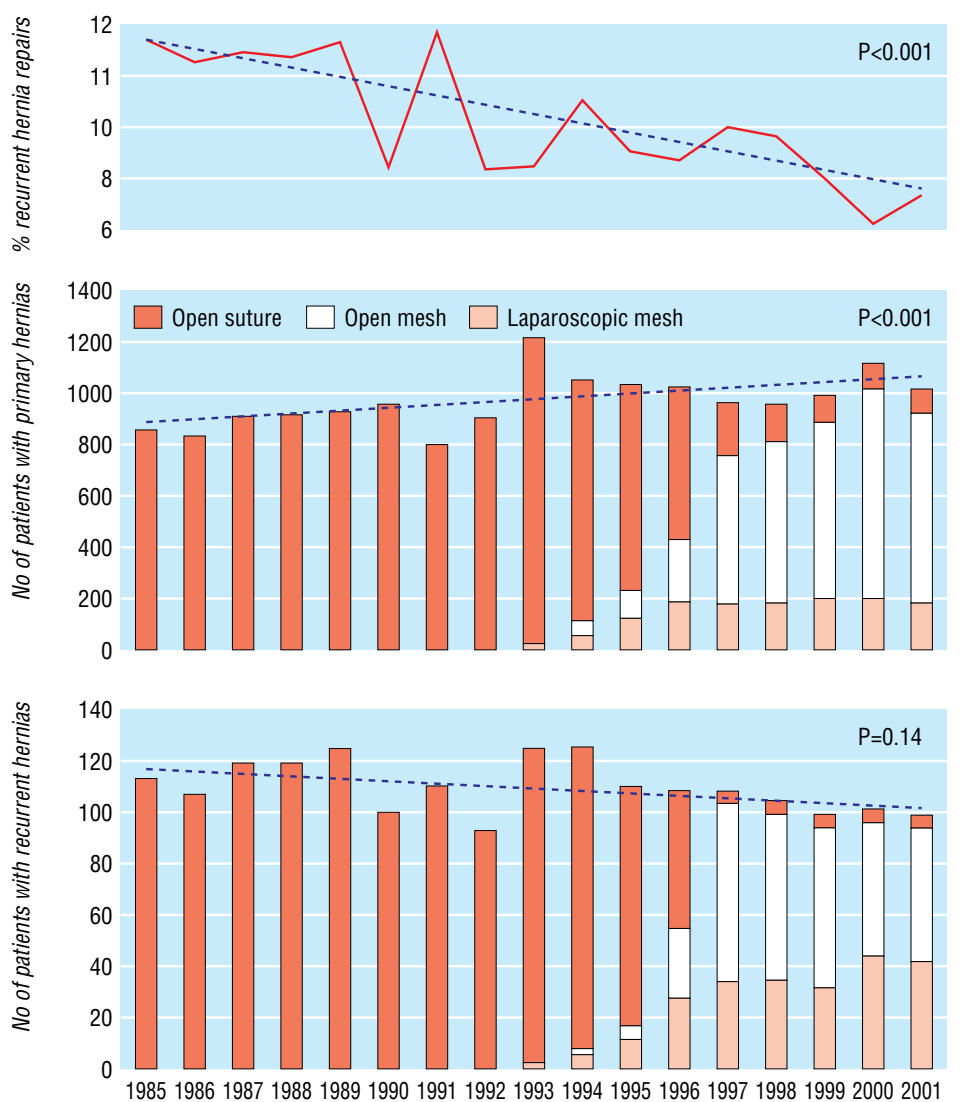

Year

Inguinal hernia repairs by open suture, open mesh, and open laparoscopic mesh techniques in southeastern Scotland from 1985 to 2001 


\section{What is already known on this topic}

Using mesh in inguinal hernia repair has increased rapidly worldwide since 1989 and is associated with low recurrence rates

\section{What this study adds}

Use of mesh in the past decade in southeastern Scotland mirrors the global trend. The annual ratio of recurrent to primary repairs has significantly decreased perhaps partly because of newer techniques but also other factors, such as better supervision and training

within one year of surgery, ${ }^{4}$ and therefore it is likely that new techniques have influenced the recurrence rates in the 17 years of this study.

We thank Stephanie C Lewis for her help and advice with the statistics.

Contributors: HDEA and SP-B designed the study. HDEA collected the data. HDEA, SGN, and SP analysed the data and wrote the initial draft. SGN and HDEA revised the draft and prepared the final manuscript. HDEA is guarantor.

Funding: None

Competing interests: None declared.

Ethical approval: Not needed.

1 Lichtenstein IL, Shulman AG, Amid PK, Montlor HM. The tension-free hernioplasty. Am J Surg 1989;157:188-93.

2 McCormack K, Scott NW, Go PM, Ross S, Grant AM, Laparoscopic techniques versus open techniques for inguinal hernia repair. Cochrane Database Syst Rev 2003(1):CD001785.

3 Schumpelick V, Treutner KH, Arlt G. Inguinal hernia repair in adults. Lancet 1994;344:375-9.

4 EU Hernia Trialists Collaboration. Mesh compared with non-mesh methods of open groin hernia repair: systematic review of randomized controlled trials. BrJ Surg 2000:87:854-9.

5 Bay-Nielsen M, Kehlet H, Strand L, Malmstrom J, Andersen FH, Wara P, et al. Quality assessment of 26304 herniorrhaphies in Denmark; a prospective nationwide study. Lancet 2001;358:1124-8.

(Accepted 31 July 2004)

doi 10.1136/bmj.38282.675556.F7

\title{
DRUG POINTS
}

\section{Sibutramine may be associated with memory impairment}

\author{
David W J Clark, Mira Harrison-Woolrych
}

Intensive Medicines

Monitoring

Programme

(IMMP), $\mathrm{New}$

Zealand

Pharmacovigilance

Centre, Department

of Preventive and

Social Medicine,

University of Otago,

Dunedin, New

Zealand

David W J Clark

senior research fellow,

IMMP

Mira

Harrison-Woolrych

director, IMMP

Correspondence to:

M Harrison-

Woolrych, PO Box

913, Dunedin, New

Zealand.

Mira.harrison-

woolrych@stonebow. otago.ac.nz

BMJ 2004;329:1316
Sibutramine (Reductil, Abbott), a serotonin and noradrenaline reuptake inhibitor is widely used in the management of obesity. The Intensive Medicines Monitoring Programme in New Zealand has received a report of amnesia in a 39 year old woman taking sibutramine $10 \mathrm{mg}$ daily. Memory impairment became evident one month after starting sibutramine and progressively worsened until her daily activities were severely affected. She also experienced increased frequency of migraines associated with neck stiffness. Within two weeks of stopping sibutramine her amnesia improved, and it had resolved completely by six weeks. Another case has been reported to the Australian Adverse Drug Reactions Advisory Committee. A 50 year old woman experienced amnesia, headache, and insomnia one day after starting sibutramine $10 \mathrm{mg}$ daily, with the symptoms resolving four days after discontinuation.

The World Health Organization's internationa database contains 33 further reports of amnesia associated with sibutramine. In 25 cases, sibutramine was the only drug suspected of causing the amnesia. Onset of amnesia was within four days of sibutramine administration in 13 cases and between 5 and 30 days after administration in four cases. Ten patients were reported to have recovered after stopping sibutramine Three patients with amnesia associated with sibutramine were also reported to have experienced a cerebrovascular event, and one of these was reported with concurrent hypertension. Seven other patients experienced symptoms possibly associated with cerebrovascular insufficiency, including confusion, dizziness, dysarthria, and abnormal vision.

Memory impairment might be part of a cerebrovascular syndrome, but sibutramine may contribute directly to amnesia. Serotonergic pathways play important roles in learning and memory, and antagonists of several 5-HT receptors prevent memory impairment. ${ }^{1}$ Drugs such as sibutramine that increase synaptic serotonin concentrations may thus contribute to memory impairment. Other serotonin reuptake inhibitors have been reported to be associated with memory loss. ${ }^{23}$

Although amnesia is listed as a reported event during postmarking surveillance of sibutramine in the US product information, ${ }^{3}$ our literature searches found no published reports of this adverse event.

We acknowledge the helpful cooperation of Patrick Purcell and Richard Hill from the Australian Adverse Drug Reactions Advisory Committee and staff of the World Health Organization's Uppsala monitoring centre.

Funding: The Intensive Medicines Monitoring Programme receives most of its funding from the New Zealand Ministry of Health. Donations towards monitoring studies are also received from some pharmaceutical companies, including Abbott, who have contributed towards the monitoring of sibutramine but have no role in analysis, interpretation, or the decision to submit signals of adverse reactions for publication.

Competing interests: None declared.

Buhot MC, Martin S, Segu L. Role of serotonin in memory impairment. Ann Med 2000;32:210-21.

Joss JD, Burton RM, Keller CA. Memory loss in a patient treated with fluoxetine. Ann Pharmacother 2003;37:1800-3.

Physicians Desk Reference (online). www.pdr.net/ (accessed 18 Nov 2004).

\section{Endpiece}

Misery

If there is technological advance without social advance, there is, almost automatically, an increase in human misery.

Michael Harrington (1928-89), American author and Democratic Socialist in The Other America (1962)

Fred Charatan, retired geriatric physician, Florida 